\title{
Origin of Germ Cells and Follicular Renewal in Adult Human Ovaries
}

Antonin Bukovsky

Laboratory of Development, Differentiation and Cancer, Department of Obstetrics and Gynecology, The University of Tennessee Graduate School of Medicine, 1924 Alcoa Hwy, Knoxville, TN 37920, USA

The origin of oocytes and primary follicles in ovaries of adult mammalian females has been a matter of dispute over one hundred years and resulted in the dogma that all oocytes in adult mammalian females persist from the fetal period of life. However, from a phylogenetic viewpoint, it seems contradictory that mammalian females, including humans, would evolve a uniquely retrogressive reproductive mechanism, whereby they are required to preserve their gametes from the fetal period for up to several decades. Mitotically active germ cells have been reported in ovaries of adult prosimian primates [1] and mice [2], and we have shown that mesenchymal cells in ovarian tunica albuginea differentiate into surface epithelium, a source of germ cells entering vessels and contributing to the follicular renewal in adult human females [3]. These reports represent challenges to the established dogma on fetal origin of mammalian eggs $[4,5]$.

Our observations indicate that cytokeratin $18+$ mesenchymal cells residing in the ovarian tunica albuginea (TA) are progenitors for ovarian surface epithelium (SE) [3] (Fig. 1A). The SE cells are a bipotent source of germ cells and primitive granulosa cells, which differentiate sequentially and de novo in the adult human ovary. This contrasts with a continuous preservation of the germline stem cells in male individuals and female mice $[2,6]$. New primary follicles are formed by assembly of intravascular oocytes with nests of primitive granulosa cells in the lower ovarian cortex (panels B-D). The number of newly formed primary follicles in adult human ovaries appears to be determined by the number of developing nests of primitive granulosa cells and not developing oocytes, since excessive oocytes were found to degenerate in medullary vessels (panel E). Formation of new primary follicles throughout the reproductive period may compensate for the well documented atresia of a significant proportion of the follicular pool. This may contribute to the selection of the best possible oocytes and ensure preservation of the relatively constant number of primary follicles found in human females between 18 and 38 years of life (panel F). It appears that during the reproductive period in human females, the pool of primary follicles does not represent a static but a dynamic population of differentiating and regressing structures. Differentiation of primitive granulosa and germ cells from the bipotent mesenchymal cell precursors of TA in adult human ovaries may represent a most sophisticated mechanism created during the evolution of female reproduction [3]. Recently, we also observed that oocytes and granulosa cells differentiate in the ovarian surface epithelium cultures derived from adult human ovaries [7].

\section{REFERENCES}

[1] J. M. Ioannou, J. Embryol. Exp. Morphol. 17 (1968) 139.

[2] J. Johnson et al., Nature 428 (2004) 145.

[3] A. Bukovsky et al., Reprod. Biol. Endocrinol. 2 (2004) 20.

[4] F. W. Bazer, Reprod. Biol. Endocrinol. 2 (2004) 28.

[5] R. G. Gosden, Hum. Reprod. Update 10 (2004) 193.

[6] A. C. Spradling, Nature 428 (2004) 133.

[7] A. Bukovsky and M. Svetlikova, Nature (2005) (submitted February 3).

[8] This work was supported by the Physicians' Medical Education \& Research Foundation (\# 679). The aid of Marta Svetlikova, M.S., and Nirmala B. Upadhyaya, M.D., is gratefully acknowledged. 

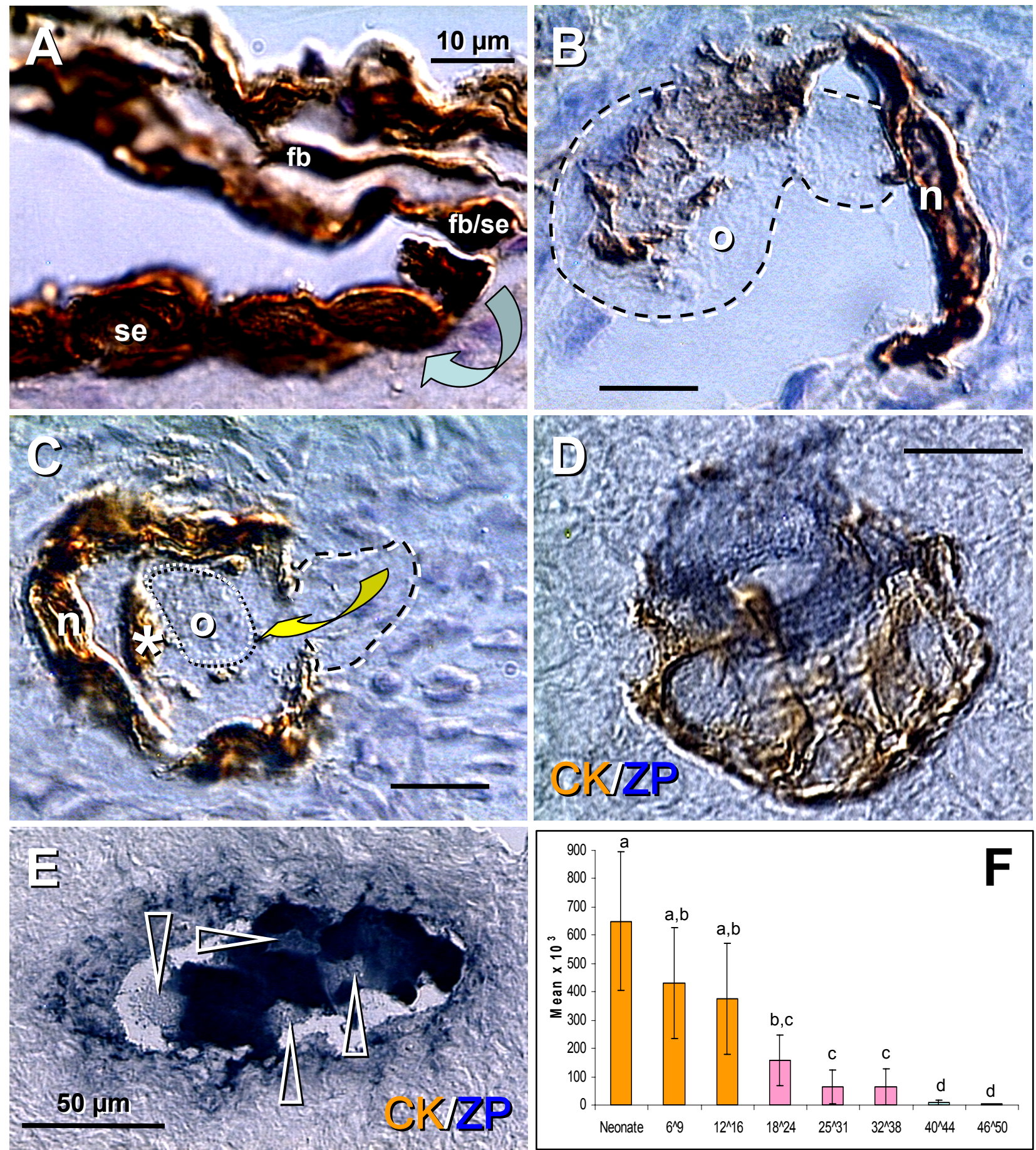

Fig. 1. CK18 (A-C) and CK/zona pellucida (ZP) staining (D and E). A) Transition of TA fibroblasts (fb) into SE cells (se). B) The intravascular oocyte (o) is "catched" by epithelial nest (n). C) Formation of Balbiani body (asterisk) during oocyte/nest assembly. D) Oocyte (blue)/nest (brown) assembly. E) Degenerating ZP+ oocytes in a medullary vessel (unstained nuclei indicated by arrowheads). F) Number of primary follicles in human ovaries. Bars in $A-D=10 \mu \mathrm{m}$. For more details see http://www.rbej.com/content/2/1/20 